\title{
ESTUDO DE MODELAGEM MOLECULAR DE COMPLEXOS FERRIPROTOPORFIRINA-IX E QUINOLINOCARBINOLAMINAS ANTIMALÁRICAS: PROPOSTA DE UM FARMACÓFORO
}

\author{
Thais Horta Álvares da Silva* e Marcelo Tavares de Oliveira \\ Departamento de Produtos Farmacêuticos, Faculdade de Farmácia, Universidade Federal de Minas Gerais, Av. Antônio Carlos, \\ 6627, 31270-901 Belo Horizonte - MG \\ Hélio Ferreira dos Santos \\ Departamento de Química, Instituto de Ciências Exatas, Universidade Federal de Juiz de Fora, 36036-330 Juiz de Fora - MG \\ Alaíde Braga de Oliveira \\ Fundação Ezequiel Dias, Rua Conde Pereira Carneiro, 80, 30510-010 Belo Horizonte - MG \\ Wagner Batista De Almeida \\ Departamento de Química, Instituto de Ciências Exatas, Universidade Federal de Minas Gerais, Av. Antônio Carlos, 6627, \\ 31270-901 Belo Horizonte - MG
}

Recebido em 22/4/04; aceito em 20/8/04; publicado na web em 4/2/05

\begin{abstract}
MOLECULAR MODELING STUDY OF COMPLEXES BETWEEN FERRIPROTOPORPHYRIN IX AND ANTIMALARIAL 4-QUINOLINECARBINOLAMINES: A PROPOSAL OF PHARMACOPHORE. Quinine and quinidine are well-known 4quinolinecarbinolamines that exhibit antimalarial activity, but, in contrast, their epimers 9-epiquinine and 9-epiquinidine are almost inactive. Literature data are conflicting in describing the 4-quinolinecarbinolamine interaction mode with the molecular target, the ferriprotoporphyrin IX [Fe(III)PPIX]. In the present paper, a pharmacophore is proposed based on the binding of the nonaromatic nitrogen to the iron atom. The 4-quinolinecarbinolamine antimalarials were superimposed on the pharmacophore under consideration and complexes with Fe(III)PPIX were constructed. Conformational analyses of the complexes were performed applying the MM+ molecular mechanics method. The analysis of the complexes showed that the proposed ligand mode is possible although it does not explain the activity differences between epimers. A discussion of the structural aspects is also provided.
\end{abstract}

Keywords: antimalarial; quinine; pharmacophore.

\section{INTRODUÇÃO}

A malária é a mais disseminada de todas as doenças infecciosas. A doença leva o indivíduo parasitado a uma anemia intensa e, em conseqüência, prejudica sua produtividade no trabalho. A doença pode ainda ter decurso fatal. Atualmente, $40 \%$ da população mundial, principalmente aquelas que vivem nos países mais pobres, vivem sob o risco de contrair malária. A málaria foi eliminada, no século XX, em vários países de clima temperado. Entretanto, ainda é encontrada em regiões tropicais e subtropicais do mundo e causa mais de 300 milhões de casos agudos e pelo menos um milhão de mortes por $a^{1}{ }^{1}$. A maioria das mortes causadas por malária ocorre em crianças da África. O Brasil está entre os maiores focos de malária no mundo ${ }^{2}$.

Os agentes etiológicos da malária humana são protozoários do gênero Plasmodium, compreendendo quatro espécies: P. falciparum, $P$. vivax, $P$. ovale e $P$. malariae. Os vetores são mosquitos do gênero Anopheles $^{3}$. No homem, o ciclo biológico inicia-se com a picada do mosquito infectado que injeta na corrente sangüínea o parasita. $\mathrm{O}$ Plasmodium invade as células do fígado e ali se multiplica. Estas células são rompidas e o parasita cai na corrente sangüínea, indo infectar os eritrócitos. Dentro dos eritrócitos os parasitas se desenvolvem, dando origem a merozoítos que são liberados no sangue com lise destas células. A destruição cíclica dos eritrócitos e liberação do pigmento malárico levam ao acesso malárico.

Do córtex do tronco e da raiz de espécies de Cinchona sp (quina), árvores naturais dos Andes, são extraídos mais de vinte alcalóides,

*e-mail: thais@farmacia.ufmg.br dentre os quais dois pares de diastereoisômeros, quinina e quinidina, cinchonina e cinchonidina, têm atividade antimalárica. Tais alcalóides existem também na forma epimérica: epiquinina e epiquinidina, epicinchonina e epicinchonidina, que são inativos. Na Tabela 1 estão relatadas as atividades in vitro (IC50) da quinina, quinidina, epiquinina e epiquinidina frente a cepas de $P$. falciparum suceptíveis e resistentes à cloroquina ${ }^{4}$. Até a 2aㅡ Guerra Mundial, a quinina (Figura 1) era o único agente antiparasitário eficaz frente à malária. Após a introdução de derivados sintéticos, a quinina foi descartada. Entretanto, com o aparecimento de cepas resistentes de Plasmodium, este fármaco foi reintroduzido. Ele tem sido usado em infecções por $P$. falciparum resistente à cloroquina e no tratamento da malária severa $^{5}$. A quinidina mostrou-se um antimalárico mais eficaz e mais tóxico que a quinina, em testes in vitro e in vivo ${ }^{4}$.

Tabela 1. Valores de atividade in vitro $\left[\mathrm{IC}_{50}(\mathrm{nM})\right]$ frente a $P$. falciparum usando clone Sierra Leone D-6 sensível à cloroquina e clone Indochina W-2 resistente à cloroquina, obtidos da ref. 4

\begin{tabular}{lcccc}
\hline & Quinina & Quinidina & Epiquinina & Epiquinidina \\
\hline $\mathrm{IC}_{50}$ no clone D-6 & 29,3 & 13,4 & 3471,0 & 2700,0 \\
$\mathrm{IC}_{50}$ no clone W-2 & 103,2 & 151,7 & 1179,0 & 1024,0 \\
\hline
\end{tabular}

Durante a 2 ${ }^{\mathrm{a}}$ Guerra Mundial, as forças aliadas inauguraram um programa de pesquisa de antimaláricos, onde foram desenvolvidos vários fármacos, notavelmente a cloroquina (Figura 1) e a primaquina. A cloroquina, uma 4-aminoquinolina, tornou-se, então, o fármaco esquizonticida sangüíneo mais usado ${ }^{6}$. 


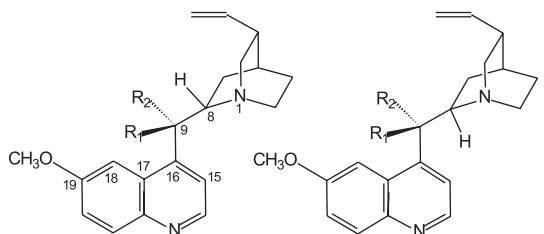

Quinina $\quad \mathrm{R}_{1}=\mathrm{OH}, \mathrm{R}_{2}=\mathrm{H} \quad$ Quinidina $\quad \mathrm{R}_{1}=\mathrm{H}, \mathrm{R}_{2}=\mathrm{OH}$

Epiquinina $\mathrm{R}_{1}=\mathrm{H}, \mathrm{R}_{2}=\mathrm{OH}$ Epiquinidina $\mathrm{R}_{1}=\mathrm{OH}, \mathrm{R}_{2}=\mathrm{H}$

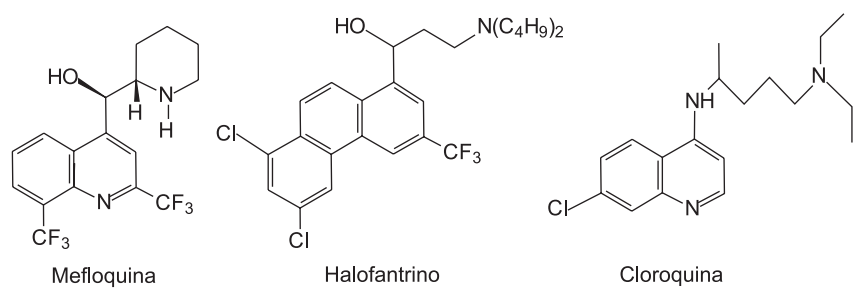

Figura 1. Estruturas de fármacos antimaláricos e análogos

No período de 1963 a 1986, devido ao aparecimento, no Vietnã, de malária resistente à cloroquina, as forças armadas dos Estados Unidos realizaram um programa intensivo de pesquisa de novos agentes antimaláricos, em que foram ensaiados mais de 300.000 compostos. Foram selecionados dois compostos com atividade contra cepas resistentes ao P. falciparum: a mefloquina, um quinolinometanol, e o halofantrino, um fenantrenometanol ${ }^{7}$ (Figura 1). A mefloquina tem dois carbonos assimétricos e é usada clinicamente como uma mistura racêmica dos isômeros eritro, que são igual ou ligeiramente mais ativos que os isômeros treo $^{8}$. O halofantrino, com apenas um carbono assimétrico, é usado clinicamente como racemato.

O planejamento racional de novos fármacos antimaláricos requer o conhecimento preciso do mecanismo de ação destes fármacos. Existem algumas evidências que a interação entre os antimaláricos esquizonticidas sangüíneos com o grupo heme [ferriprotoporfirina IX (Fe(III)PPIX)- Figura 2] está envolvida na toxicidade destes fármacos ao parasita intraeritrocítico. Vários experimentos in vitro estabeleceram que fármacos antimaláricos quinolínicos agem por interferência na cristalização da hemozoína9 ${ }^{9}$. É consenso que estes fármacos inibem a formação da hemozoína. Persiste, entretanto, uma divergência sobre como isto ocorre ${ }^{10}$.

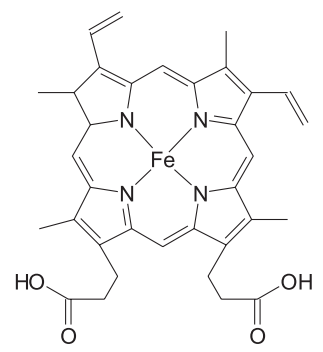

Figura 2. Estrutura da Fe(III)PPIX

Os parasitas degradam cerca de $75 \%$ da hemoglobina dos eritrócitos e a utilizam como fonte alimentar. A hemoglobina é importada para dentro de um compartimento acídico do parasita, conhecido como vacúolo alimentar, e é quebrada por enzimas proteolíticas, chamadas plasmecinas, em peptídeos que, posteriormente, são degradados a aminoácidos ${ }^{10}$. O resíduo livre heme ou ferriprotoporfirina IX (Fe(III)PPIX) é tóxico ao parasita. A Fe(III)PPIX é polimerizada formando um composto inerte, insolúvel e não tóxico ao parasita, o pigmento malárico hemozoína.

Foi demonstrado química, espectroscópica e cristalograficamente que o pigmento malárico hemozoína é idêntico à $\beta$-hematina sintética, cuja estrutura foi determinada usando técnicas de "simulated annealing" para analisar dados de difração, obtidos por radiação síncroton ${ }^{11}$. No cristal de $\beta$-hematina, as moléculas de Fe(III)PPIX estão ligadas, formando dímeros, por meio de ligações recíprocas ferro-carboxilato de uma das cadeias laterais propiônicas. Estes dímeros formam cadeias mantidas por ligações de hidrogênio (Figura 3).

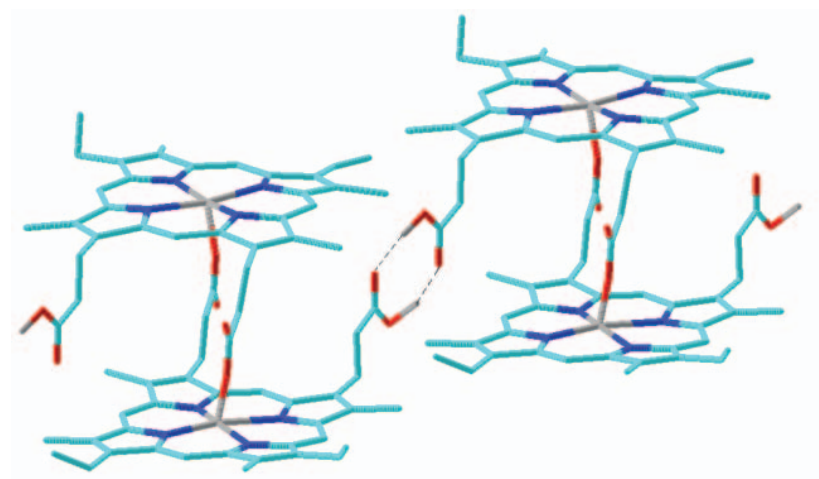

Figura 3. Representação da estrutura cristalográfica da $\beta$-hematina, adaptada da ref. 11

Slater e Cerami ${ }^{12}$ sugeriram que a reação de formação da hemozoína no parasita é catalisada por uma enzima, que seria inibida pelos antimaláricos quinolínicos. Esta conclusão foi baseada nas condições extremas que, aparentemente, seriam requeridas para a formação por síntese da $\beta$-hematina e na observação que um extrato de membrana plasmodial aparentemente catalisa esta formação. Entretanto, Dorn e colaboradores ${ }^{13}$ encontraram que a formação de $\beta$ hematina é independente de proteínas e sugeriram que ela é autocatalítica, sendo que o agente catalítico no extrato do parasita é, de fato, a própria hemozoína.

Uma outra hipótese é que os antimaláricos quinolínicos podem inibir a formação de hemozoína, pela interação direta com a $\mathrm{Fe}(\mathrm{III})$ PPIX. Warhurst ${ }^{14}$ demonstrou espectroscopicamente que antimaláricos quinolínicos formam, em solução benzênica, complexos com a Fe(III)PPIX e que o epímero inativo da quinina, a 9epiquinina, não forma o complexo. Egan e colaboradores ${ }^{15}$ demonstraram que a cloroquina, quinina e amodiaquina são capazes de inibir a polimerização espontânea da Fe(III)PPIX em solução acídica acética, enquanto a 9-epiquinina e outras substâncias inativas na fase eritrocítica da malária não são. Dorn e colaboradores ${ }^{16}$ também relataram que a formação de $\beta$-hematina é inibida por antimaláricos quinolínicos sob condições mais brandas, que se aproximam mais daquelas que são esperadas in vivo.

Portanto, o efeito primário dos fármacos antimaláricos que atuam na fase eritrocítica da doença é a ligação com a Fe(III)PPIX e a inibição da sua polimerização para formação da hemozoína. Secundariamente, a Fe(III)PPIX e o complexo FE(III)PPIX-fármaco acumulam-se e ficam disponíveis para exercer seus efeitos tóxicos ${ }^{17}$. A base precisa para os efeitos tóxicos da Fe(III)PPIX livre e seus complexos com antimaláricos quinolínicos no parasita não está completamente estabelecida ${ }^{10}$. Uma das hipóteses é que esta toxicidade resulta da atividade peroxidativa da Fe(III)PPIX e dos complexos $\mathrm{Fe}(\mathrm{III}) \mathrm{PPIX}$-fármaco sobre os lipídeos da membrana ${ }^{18}$. Outra hipótese é que a Fe(III)PPIX lisa o parasita via um mecanismo colóidoosmótico, possivelmente pela inibição da manutenção do gradiente de cátions ${ }^{10}$.

Por meio do estudo do espectro eletrônico de complexos da $\mathrm{Fe}(\mathrm{III}) \mathrm{PPIX}$ com fármacos antimaláricos, Warhurst ${ }^{14}$ propôs que as quinolinocarbinolaminas ativas formavam ligação com o ferro por 
meio do nitrogênio do anel não aromático e por interações do tipo $\pi-\pi$ entre os anéis aromáticos. Constantinides e Satterlee ${ }^{19}$, por meio de estudos de $\mathrm{RMN}{ }^{13} \mathrm{C}$ de complexos heme-quinina, encontraram evidências da coordenação do ferro da Fe(III)PPIX com o oxigênio carbinólico da quinina e também de interações do tipo $\pi$ - $\pi$ entre os anéis aromáticos da quinina e da Fe(III)PPIX .

A espectroscopia de Mössbauer foi utilizada por Adams e colaboradores ${ }^{20}$ na elucidação da natureza do ambiente do ferro na hemozoína e em complexos Fe(III)PPIX -antimaláricos. Foi proposto que os antimaláricos do tipo 4-quinolinocarbinolamina, como a quinina e a amodiaquina, se complexariam por meio de seus oxigênios carbinólicos com o ferro e por interações do tipo $\pi-\pi$ entre os anéis aromáticos.

Marques e colaboradores ${ }^{21}$ realizaram o estudo espectroscópico da complexação de um derivado do citocromo C (o hemeoctapeptídeo que tem na sua estrutura a Fe(III)PPIX com a quinina e a 9-epiquinina). Foi mostrado que ambas moléculas formavam complexos de coordenação com o ferro por meio do átomo de oxigênio carbinólico. A habilidade da 9-epiquinina em formar o complexo foi considerada uma surpresa, em função da sua inabilidade em inibir a formação da hemozoína e de se ligar à Fe(III)PPIX em outros experimentos. A razão desta discrepância não foi explicada. Entretanto, os pesquisadores concluíram que a ligação de quinolinas com a Fe(III)PPIX é um requerimento necessário mas não suficiente para a atividade antimalárica. Nos estudos de modelagem molecular ficou confirmado que tanto a quinina quanto a epiquinina são capazes de formar complexo com a Fe(III)PPIX, via oxigênio carbinólico ${ }^{21}$. Portanto, este tipo de interação não explica a diferença de atividade entre a quinina e a epiquinina.

Um estudo computacional detalhado de uma série de 4-quinolinocarbinolaminas, dentre elas a mefloquina, com o objetivo de correlacionar aspectos eletrônicos com a atividade antimalárica, foi descrito por Bhattachajee e Karle ${ }^{22}$. O estudo mostrou que a coordenação entre o grupo hidroxila da quinina (e 9-epiquinina) com o Fe(III) é possível, com considerável flexibilidade do complexo. Foi também relatada a modelagem molecular da interação de 4-aminoquinolinas com a Fe(III)PPIX ${ }^{23}$. Neste estudo foram calculadas as energias de interação entre os fármacos e a Fe(III)PPIX. Nas estruturas obtidas para os complexos amodiaquina- e tebuquina-Fe(III)PPIX encontraram-se interações cofaciais entre o anel quinolínico e a Fe(III)PPIX.

Os estudos de complexação com a Fe(III)PPIX são problemáticos devido à tendência de agregação da Fe(III)PPIX em meio aquoso e, muitas vezes, levam a resultados difíceis de serem interpretados. Essencialmente dois métodos têm sido usados para contornar este problema. Os estudos são realizados em meio não aquoso, solventes mistos ou em soluções detergentes, ou substituindo o heme por heme-peptídeos ou ferroporfirinas com pouca tendência a se agregarem em solução aquosa. Estudos em condições diferentes descritos na literatura têm levado a resultados contraditórios.

Egan e colaboradores ${ }^{24}$ determinaram a constante de ligação entre quinolinas com a Fe(III)PPIX monomérica em solução a $40 \%$ de dimetilsulfóxido em água. Em pH 7,5, os valores encontrados para o $\log \mathrm{K}$ foram 4,10 $\pm 0,02$ para a quinina e 4,04 $\pm 0,03$ para epiquinina. As interações entre a quinina e epiquinina com a Fe(III)PPIX são mantidas fortes em $\mathrm{pH} 5,6, \mathrm{pH}$ aproximado do vacúolo digestivo do parasita malárico. A interação da 9-epiquinina com a Fe(III)PPIX é inesperada e contraditória aos relatos anteriores, em que a 9epiquinina não se complexa com a Fe(III)PPIX em benzeno ${ }^{14}$.

A análise conformacional dos antimaláricos quinina ${ }^{25}$ e quinidina $^{26}$ e dos seus epímeros inativos, epiquinina e epiquinidina ${ }^{27}$ foi realizada pelo nosso grupo. A análise conformacional destes alcalóides teve como objetivo correlacionar aspectos estruturais com a atividade antimalárica.
Nos trabalhos relatados na literatura ${ }^{14,19-21}$ foram utilizados diferentes técnicas, tanto na obtenção quanto na caracterização dos complexos Fe(III)PPIX -fármaco. As diferentes conclusões a respeito do tipo de interação que ocorre entre os fármacos e a Fe(III)PPIX podem ser atribuídas às diferentes metodologias utilizadas nestes trabalhos. Entretanto, quando se considera que a interação ocorre via átomo de oxigênio carbinólico do fármaco, tanto a quinina (ativa) quanto a epiquinina (inativa) são capazes de se ligar à Fe(III)PPIX, como foi demonstrado por estudos de modelagem molecular ${ }^{21}$. Desta forma, no presente estudo, uma nova proposta de farmacóforo para os fármacos do tipo 4-quinolinocarbinolamina é apresentada, considerando que estes fármacos se ligam ao ferro por meio da amina não aromática. Considerou-se ainda que deveria existir uma interação $\pi-\pi$ entre os anéis aromáticos porfirínico da Fe(III)PPIX e quinolínico dos fármacos antimaláricos.

No farmacóforo proposto ${ }^{28}$ (Figura 4), o plano do anel quinolínico deve ficar perpendicular ao par de elétrons livres do nitrogênio quinuclidínico e ainda deve haver uma região livre de impedimento estéreo para permitir a ligação do nitrogênio ao ferro e a interações $\pi$ - $\pi$ entre o anéis aromáticos quinolínico (do fármaco) e porfirínico (da Fe(III)PPIX).

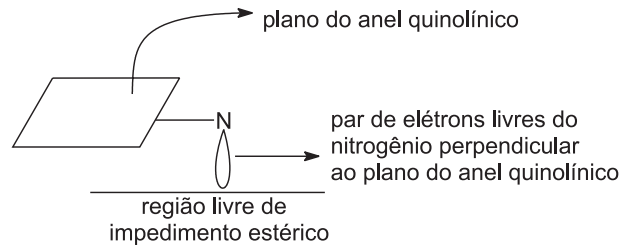

Figura 4. Representação do farmacóforo proposto para 4-quinolinocarbinolaminas $^{27}$

\section{PARTE EXPERIMENTAL}

As coordenadas atômicas da hemoglobina humana foram obtidas no "Protein Data Bank"29. Foi selecionado um dos quatro grupos heme (Fe(III)PPIX) presentes na molécula da hemoglobina, ao qual estava ligado um resíduo de histidina, e o restante da molécula foi excluído do arquivo. Do resíduo de histidina foi mantido apenas o átomo de nitrogênio ligado ao átomo de ferro, com o objetivo de servir de referência para a ligação das moléculas das quinolinocarbinolaminas.

As coordenadas das conformações de menor energia das quinolinocarbinolaminas foram obtidas das refs. 25 a 27. Para a sobreposição ao farmacóforo proposto foram usadas as conformações de menor energia encontradas para a quinina $(\mathrm{QN})$, quinidina (QD), epiquinina (EQN) e epiquinidina (EQD).

No farmacóforo proposto o par de elétrons livres do nitrogênio do anel quinuclidínico deve ficar em posição perpendicular ao plano do anel aromático quinolínico (Figura 4). Foram realizadas rotações nos ângulos $\alpha(\mathrm{N} 1, \mathrm{C} 8, \mathrm{C} 9, \mathrm{C} 16)$ e $\beta(\mathrm{C} 8, \mathrm{C} 9, \mathrm{C} 16, \mathrm{C} 17)$ (Figura 1), obtendo-se, para cada uma das quatro moléculas estudadas, quatro conformações sobreponíveis ao farmacóforo. Nas Figuras 5 e 6 são mostradas as projeções de Newman para as conformações da quinina e epiquinina, que se sobrepõem ao farmacóforo proposto.

As moléculas das quinolinocarbinolaminas estudadas nas conformações farmacofóricas foram posicionadas em relação à Fe(III)PPIX de maneira que o N1 ocupasse a posição do nitrogênio da histidina, e que os anéis aromáticos do heme e do fármaco ficassem paralelos. Para completar o octeto do ferro, uma molécula de água foi posicionada a $0,96 \AA$ do átomo de ferro (Figura 7). As geometrias dos complexos propostos foram otimizadas usando o méto- 
do de mecânica molecular com o campo de força $\mathrm{MM}+{ }^{30}$ implementado no programa HyperChem ${ }^{31}$. As otimizações de geometrias foram feitas utilizando o algorítimo "steepst descendent" com gradiente de $0,01 \mathrm{kcal} \AA^{-1} \mathrm{~mol}^{-1}$.
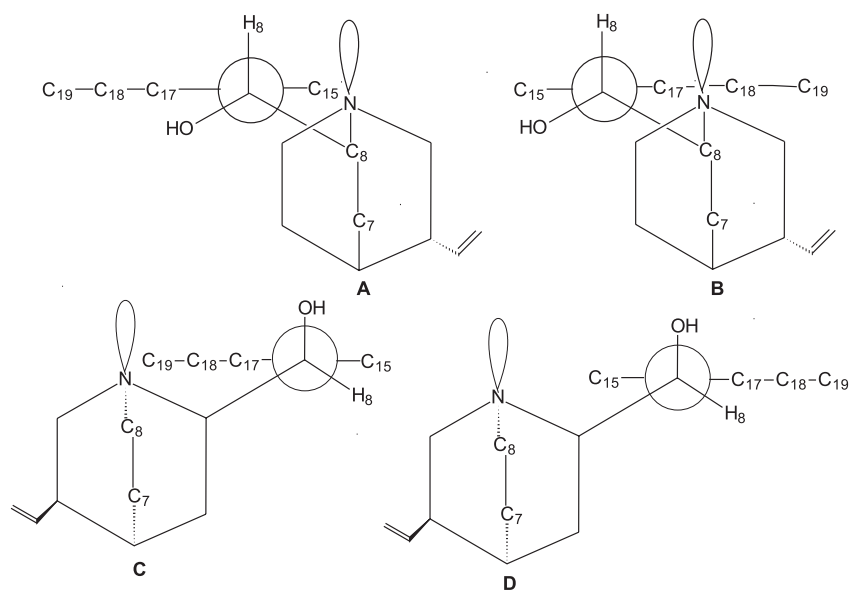

Figura 5. Representação esquemática da molécula de quinina em conformações em que o par de elétrons livres do nitrogênio quinuclidínico está perpendicular ao plano do anel quinolínico
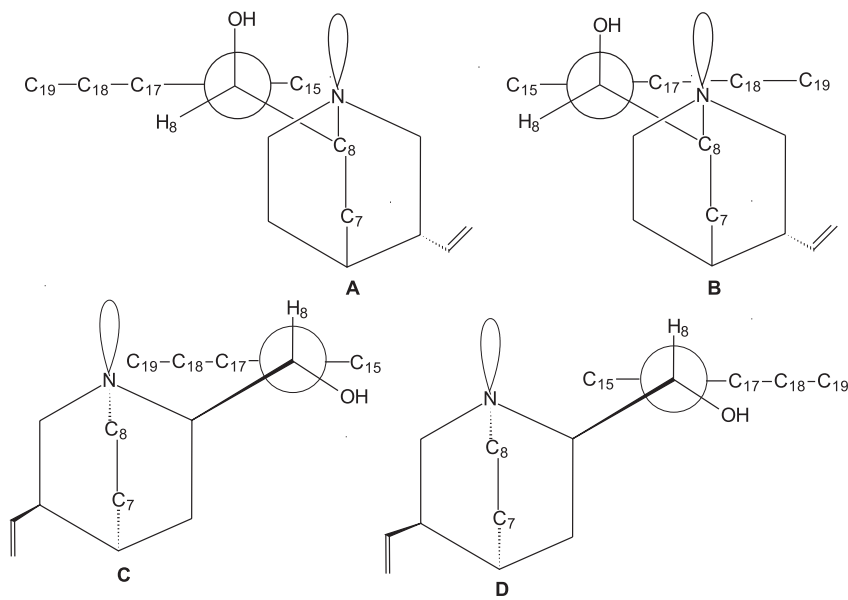

Figura 6. Representação esquemática da molécula de epiquinina em conformações em que o par de elétrons livres do nitrogênio quinuclidínico está perpendicular ao plano do anel quinolínico

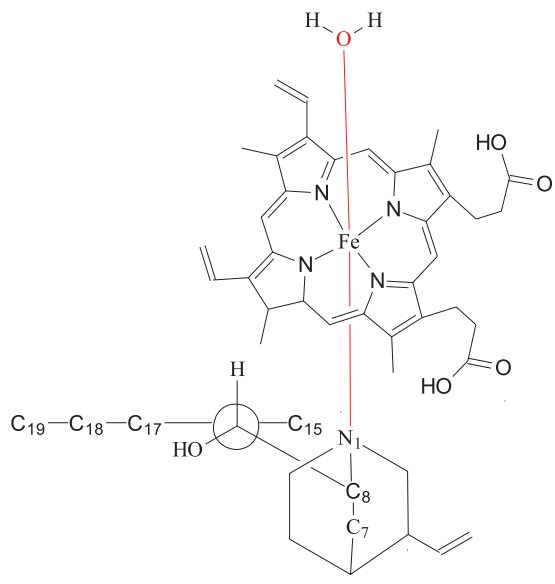

Figura 7. Representação esquemática do complexo Fe(III)PPIX-águaquinina
Cada um dos complexos quinolinocarbinolamina-Fe(III)PPIXágua obtidos foi submetido a rotações de $30^{\circ}$ em torno do ângulo torsional [N-Fe-N1-C] com o objetivo de se encontrar, no complexo, a melhor posição relativa entre a molécula de quinolinocarbinolamina e o grupo heme. A energia estérica de cada uma das conformações foi calculada. A geometria de menor energia encontrada serviu de ponto de partida para rotações com ângulos menores. As geometrias encontradas na busca conformacional foram novamente otimizadas usando o método de mecânica molecular MM+, algorítimo "steepst descendent" e gradiente de $0,01 \mathrm{kcal} \AA^{-1} \mathrm{~mol}^{-1}$.

\section{RESULTADOS E DISCUSSÃO}

Para cada uma das quinolinocarbinolaminas estudadas (QN, QD, EQN e EQD) foram encontradas quatro conformações que se sobrepõem ao farmacóforo proposto (Figura 4). Com cada uma delas foi formado um complexo com a Fe(III)PPIX, que foi submetido à análise conformacional levando a quatro estruturas cujos dados estão mostrados na Tabela 2.

Tabela 2. Dados estruturais (ângulos torsionais $\alpha$ (N1,C8,C9,C16) e $\beta(C 8, C 9, C 16, C 17)$, em graus) e de energia estérica $\mathrm{MM}+(\mathrm{em}$ $\mathrm{kcal} \mathrm{mol}^{-1}$ ) calculados na fase gasosa para os confôrmeros estáveis localizados na superfície de energia potencial dos complexos das moléculas de quinina, quinidina, epiquinina e epiquinidina com a Fe(III)PPIX

\begin{tabular}{lcrc}
\hline Conformação & Ângulo alfa & Ângulo beta & EE $\left(\mathrm{kcal} \mathrm{mol}^{-1}\right)$ \\
\hline \multicolumn{4}{c}{ QUININA } \\
\hline HEME-QNA & 153 & -175 & 124,812 \\
HEME-QNB & 149 & 10 & 125,396 \\
HEME-QNC & 152 & -179 & 121,838 \\
HEME-QND & -59 & 180 & 143,629 \\
\hline \multicolumn{5}{c}{ QUINIDINA } \\
\hline HEME-QDA & -151 & -4 & 128,283 \\
HEME-QDB & -153 & 175 & 125,037 \\
HEME-QDC & 66 & 172 & 140,208 \\
HEME-QDD & 131 & -116 & 133,615 \\
\hline \multicolumn{5}{c}{} \\
\hline HEME-EQNA & 148 & -164 & 129,314 \\
HEME-EQNB & 145 & 189 & 132,447 \\
HEME-EQNC & -117 & 108 & 131,530 \\
HEME-EQND & -67 & 179 & 140,551 \\
\hline \multicolumn{5}{c}{ EPIQUININA } \\
\hline HEME-EQDA & -141 & -18 & 133,853 \\
HEME-EQDB & -157 & 2 & 124,606 \\
HEME-EQDC & 116 & -111 & 132,548 \\
HEME-EQDD & 70 & 179 & 140,244 \\
\hline
\end{tabular}

As diferentes estruturas obtidas para o complexo Fe(III)PPIX...fármaco (sendo o fármaco representado por quinina, quinidina, epiquinina ou epiquinidina) são representadas nas Figuras 8 a 11.

Na sobreposição das quinolinocarbinolaminas (QN, QD, EQN e EQD) ao farmacóforo, duas conformações têm o par de elétrons do $\mathrm{N}_{1}$ paralelo à ligação $\mathrm{C}_{9}-\mathrm{OH}$ (QNC e QND; QDC e QDD; EQNA e EQNB; EQDA e EQDB). As outras duas conformações de cada conjunto, i.e. QNA e QNB; QDA e QDB; EQNC e EQND; EQDC e EQDD, são mais adequadas à complexação com a Fe(III)PPIX, uma 


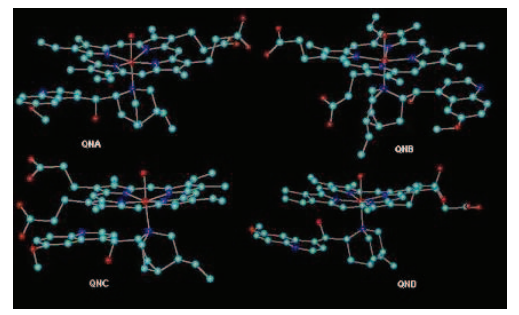

Figura 8. Estrutura dos complexos Fe(III)PPIX-quinina-água (os hidrogênios foram ocultados)

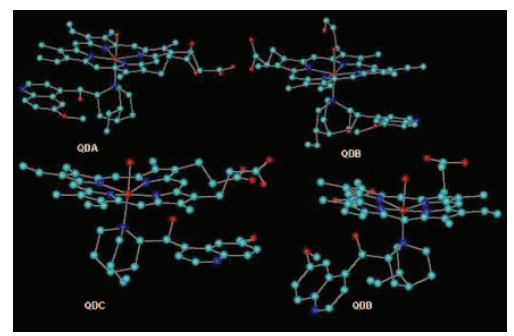

Figura 9. Estrutura dos complexos Fe(III)PPIX-quinidina-água (os hidrogênios foram ocultados)

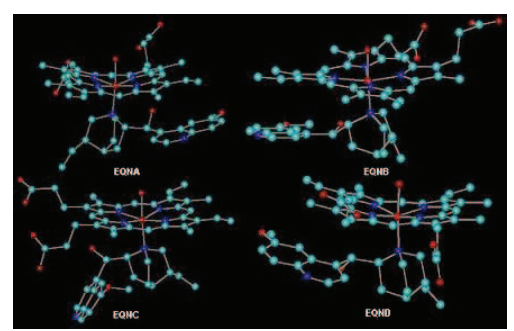

Figura 10. Estrutura dos complexos Fe(III)PPIX-epiquinina-água (os hidrogênios foram ocultados)

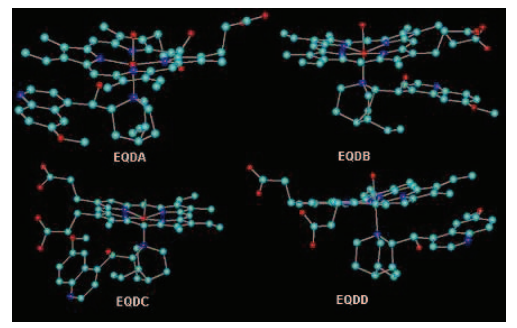

Figura 11. Estrutura dos complexos Fe(III)PPIX-epiquinidina-água (os hidrogênios foram ocultados)

vez que a hidroxila está direcionada em posição oposta à ligação com a Fe(III)PPIX.

O complexo HEME-QNC convergiu para uma conformação semelhante ao HEME-QNA, com energia estérica ligeiramente menor. A conformação HEME-QND tem energia estérica muito maior que as conformações HEME-QNA, HEME-QNB e HEME-QNC, porque nesta conformação o anel aromático porfirínico sofreu distorções para acomodar a hidroxila da quinina, que está apontada em sua direção (ver Figura 8).

O complexo HEME-QDD sofreu, durante a otimização da geometria, mudanças conformacionais que levaram o anel quinolínico da quinidina a não ficar paralelo ao anel porfirínico da Fe(III)PPIX. A conformação HEME-QDC tem energia estérica muito maior que as conformações HEME-QDA, HEME-QDB e HEME-QDD, porque nesta conformação o anel aromático porfirínico sofreu, como no exemplo anterior, distorções para acomodar a hidroxila da quinidina, que está apontada em sua direção (ver Figura 9).

Esperava-se que as conformações EQNC e EQND, por não apresentarem a hidroxila apontada na direção do par de elétrons do $\mathrm{N}_{1}$, levassem a complexos com menor energia estérica. Entretanto, os complexos HEME-EQNC e HEME-EQND sofreram, durante a otimização da geometria, mudanças conformacionais que levaram o anel quinolínico da epiquinina a não ficar paralelo ao anel porfirínico da Fe(III)PPIX (ver Figura 10). De forma análoga, era esperado que as conformações EQDC e EQDD fossem mais adequadas à ligação com a Fe(III)PPIX, tendo em vista que nas outras conformações (EQDA e EQDB) a hidroxila poderia causar impedimento estéreo por estar voltada para o mesmo lado do par de elétrons do $\mathrm{N}_{1}$. Entretanto, a conformação HEME-EQDA tem energia estérica menor que as conformações HEME-EQDB, HEME-EQDC e HEME-EQDD. Os complexos HEME-QEQDC e HEME-EQDD sofreram, durante a otimização da geometria, rotações que levaram o anel quinolínico da epiquinidina a não ficar paralelo ao anel porfirínico, no caso de HEME-EQDC, ou a se afastar do anel porfirínico da Fe(III)PPIX, no caso do HEME-EQDD (ver Figura 11).

Quando se comparam os complexos de menor energia de cada uma das moléculas (HEME-QNC, HEME-QDB, HEME-EQNA e HEME-EQDB) verifica-se que nos complexos com a epiquinina e epiquinidina o anel porfirínico está mais deformado para acomodar a hidroxila, que aponta para o seu lado e que a distância Fe-N quinuclidínico é um pouco maior que nos complexos com a quinina e quinidina, o que afasta os anéis quinolínico e porfirínico.

Já foi inferido, por estudos dos espectros de ressonância magnética nuclear ${ }^{19}$, que é o grupo hidroxila da quinina que coordena o ferro da Fe(III)uroprotoporfirina I e Fe(III)uroemina I. Entretanto a interação da hidroxila com o ferro III somente seria possível se o pKa deste grupo fosse extraordinariamente baixo, ou que ele se mantivesse protonado quando coordenado ao ferro, ou ainda que a constante de dissociação decrescesse fortemente com o $\mathrm{pH}$, o que não ocorre, como foi demonstrado por Egan e colaboradores ${ }^{24}$. Uma outra possibilidade é que essa coordenação tivesse uma contribuição negligenciável para a estabilidade do complexo. A possibilidade de coordenação com o nitrogênio não foi aventada pelos autores. Entretanto, quando se analisam os dados de RMN dos complexos de quinina e uroemina I e uroporfirina I, apresentados por Constantinidis e Satterlee ${ }^{19}$, verifica-se que a variação dos valores de deslocamento químico observada para os hidrogênios ligados aos carbonos ligados ao nitrogênio é maior que aquela observada para o hidrogênio ligado ao carbono ligado à hidroxila, o que pode ser indicativo de uma complexação via nitrogênio, como estamos supondo.

A possibilidade de compostos nitrogenados complexarem-se diretamente ao ferro foi estudada por Marques e colaboradores ${ }^{32}$, por meio da determinação das constantes de equilíbrio da substituição da água coordenada ao Fe(III)-hemeoctapeptídeoperoxidase 8 (MP8) por compostos nitrogenados. A semelhança dos espectros UV/vis entre os complexos da anilina e piridina com o MP8 e o complexo amônia-MP8 indicou que estes compostos se ligam por meio da coordenação com o metal e não por interação com o anel porfirínico. Esse tipo de interação foi confirmada pela constatação que o complexo da 1-metilpiridina, que não pode atuar como ligante ao ferro(III), tem espectro UV/vis totalmente diferente dos complexos da amônia, piridina e anilina com o MP8.

A análise conformacional realizada neste trabalho confirma a possibilidade de a ligação da quinina, quinidina, epiquinina e epiquinidina com a Fe(III)PPIX ocorrer via complexação do nitrogênio quinuclidínico ao átomo de ferro e, ainda, verificam-se interações do tipo $\pi-\pi$ entre os anéis aromáticos quinolínico e porfirínico. 
Estudos das propriedades moleculares eletrônicas de uma série de 4-quinolinocarbinolaminas ${ }^{22}$ mostraram que duas regiões de potencial negativo, que ocorrem próximas ao oxigênio da hidroxila e ao nitrogênio não aromático, são fatores importantes que se correlacionam com a atividade antimalárica destas moléculas. Estes resultados são coerentes com a nossa proposta de complexação via átomo de nitrogênio. Entretanto, vários outros estudos relatados na literatura concluem por diferentes modos de complexação de antimaláricos com a Fe(III)PPIX. Simulações por dinâmica molecular de complexos de análogos da tebuquina, uma 4-aminoquinolina, com a $\mathrm{Fe}(\mathrm{III}) \mathrm{PPIX}$ mostraram a possibilidade da ligação do ferro ao nitrogênio quinolínico, além da ligação do nitrogênio da cadeia lateral carregado positivamente com os grupos carboxilato da Fe(III)PPIX ${ }^{23}$. Numa abordagem diferente, Menezes e colaboradores ${ }^{33}$ estudaram, por modelagem molecular, a interação cátion- $\pi$ do ferro com o anel aromático de antimaláricos dos tipos $1 \mathrm{H}$-pirazolo[3,4-b]piridinocarbinolamínicos e 4-quinolinocarbinolamínicos e concluíram que esta interação se correlaciona com a atividade desses compostos ${ }^{33}$. Estudos de análise conformacional e por dinâmica molecular realizados Marques e colaboradores ${ }^{21}$ mostraram que a coordenação do grupo 9-alcóxi ao ferro(III) do grupo porfirínico é possível tanto para a quinina quanto para a epiquinina, com o anel quinolínico virtualmente paralelo à periferia do anel porfirínico.

\section{CONCLUSÃO}

A proposta do farmacóforo apresentada no presente estudo mostrou uma possibilidade de interação das quinolinocarbinolaminas com o seu alvo molecular - a Fe(III)PPIX. Mediante os resultados obtidos, não se descarta a possibilidade de os fármacos antimaláricos do tipo 4-quinolinometanol se ligarem ao ferro da Fe(III)PPIX por meio do nitrogênio não aromático, entretanto estes resultados não foram suficientes para explicar a diferença de atividade antimalárica entre quinina e quinidina e seus epímeros epiquinina e epiquinidina. Estudos usando cálculos quanto-mecânicos são indispensáveis para se obter resultados mais conclusivos sobre aspectos moleculares envolvidos na ação de 4-quinolinocarbinolaminas.

\section{AGRADECIMENTOS}

A Fundação de Amparo à Pesquisa de Minas Gerais, FAPEMIG, pelo suporte financeiro. H. F. dos Santos também agradece ao CNPq e FINEP (CTINFRA-I) pela bolsa de pesquisa e recursos computacionais financiados.

\section{REFERÊNCIAS}

1. http://www.rbm.who.int, acessada em Maio 2003.

2. World Health Organization; World health statistics quaterly 1992, 31, 2.

3. Murray, M. C.; Perkins, M. E.; Ann. Rep. Med. Chem. 1996, 31, 141.

4. Karle, J. M.; Karle, I. L.; Gerena, L.; Milhous, W. K.; Antimicrob. Agents Chemother. 1992, 36, 1538.

5. Ferreira, E. I.; Rev. Farm. Bioquím. Univ. S. Paulo 1993, $1,1$.

6. Wolf, M. E.; Burger's Medicinal Chemistry: The Basis of Medicinal Chemistry, Wiley: New York, 1980; Greenwood, D.; Antimicrob. Agents Chemother. 1995, 36, 857.

7. Foley, M.; Tilley, L.; Pharmacol. Ther. 1998, 79, 55.

8. Karle, J. M.; Olmeda, R.; Gerena, L.; Milhous, W. K.; Exp. Parasitol. 1993, 76,345 .

9. Sulivan Jr., D. J.; Matile, H.; Ridley, R. G.; Goldberg, D. E.; J. Biol. Chem. 1998, 273, 31103

10. Egan, T. J.; Marques, H. M.; Coord. Chem. Rev. 1999, 190-192, 493.

11. Pagola, S.; Stephens, P. W.; Bohle, D. S.; Kosar, A. D.; Madsen, S. K.; Nature 2000, 404, 307.

12. Slater, A.; Cerami, A.; Nature 1992, 355, 167.

13. Dorn, A.; Stoffel, R.; Matile, H.; Bubendorf, A.; Ridley, R. G.; Nature 1995, $374,269$.

14. Warhurst, D. C.; Biochem. Pharmacol. 1981, 30, 3323.

15. Egan, T. P.; Ross, D. C.; Adams, P. A.; FEBS Lett. 1994, 352, 54.

16. Dorn, A.; Vippagunta, S. R.; Matile, H.; Jaquet, C.; Vennerstom, J. L.; Ridley, R. G.; Biochem. Pharmacol. 1998, 55, 727.

17. Orjih, A. U.; Ryerse, S.; Fitch, C. D.; Experientia 1994, 50, 34; Warhurst, D. C.; Ann. Tropical Medicine and Parasitology 1987, 81, 65.

18. Sugioka, Y.; Suzuki, M.; Biochim. Biophys. Acta 1991, 1074, 19.

19. Constantinidis, J.; Satterlee, J. D.; J. Am. Chem. Soc. 1988, 110, 927; Constantinidis, J.; Satterlee, J. D.; J. Am. Chem. Soc. 1988, 110, 4391.

20. Adams, P. A.; Berman, P. A. M.; Egan, T. J.; Marsh, P. J.; Silver, J.; J. Inorg. Chem. 1996, 63, 69.

21. Marques, H. M.; Voster, K.; Egan, T. J.; J. Inorg. Biochem. 1996, 64, 7.

22. Bhattacharjee, A. K.; Karle, J. M.; J. Med. Chem. 1996, 39, 4622.

23. O’Neill, P. M.; Willock, D. J.; Hawley, S. R.; Bray, P. G.; Storr, R. C.; Ward, S. A.; Park, B. K.; J. Med. Chem. 1997, 40, 437.

24. Egan, T. M.; Mavuso, W. W.; Ross, D. C.; Marques, H. M.; J. Inorg. Chem. 1997, 68, 137.

25. Silva, T. H. A.; Oliveira, A. B.; De Almeida, W. B.; Struct. Chem. 1997, 8, 95.

26. Silva, T. H. A.; Oliveira, A. B.; De Almeida, W. B.; Bioorg. Med. Chem. 1997, 5, 353.

27. Silva, T. H. A.; Oliveira, A. B.; Dos Santos, H. F.; De Almeida, W. B.; Struct. Chem. 2001, 12, 431.

28. Silva, T. H. A.; Tese de doutorado, Universidade Federal de Minas Gerais, Brasil, 1998

29. Tame, J.; Valone, B. Em http://www.pdb.ufmg.br/pdb. Código de identificação $1 \mathrm{~A} 3 \mathrm{~N}$.

30. Allinger, N. L.; J. Am. Chem. Soc. 1977, 99, 8127.

31. Hypercube, Inc; Hyperchem ${ }^{\mathrm{TM}} 6.02$ Molecular Modeling System, 2000.

32. Marques, H. M.; Byfield, M. P.; Pratt, J. M.; J. Chem. Soc., Dalton Trans. 1993, $11,1633$.

33. Menezes, C. M. S.; Sant'Anna, C. M. R.; Rodrigues, C. R.; Barreiro, E. J.; J. Mol. Struc. (Theochem) 2002, 579, 31. 